\title{
Theory and Validation of Electricity Price Issuing Program for Concentrated Meter Reading
}

\author{
Gang Wang ${ }^{1, a}$, Xiaohua Liu ${ }^{1, b}$, Xiaofei Dai ${ }^{1, c}$ and Lequn Zhang ${ }^{2, d}$ \\ ${ }^{1}$ State Grid XinJiang Electric Power Corporate, Urumchi, China \\ ${ }^{2}$ Qingdao Topscomm Communication Co., Ltd, Qingdao, China \\ a wg619@126.com, ${ }^{\mathrm{b}}$ 1173308892@qq.com, ${ }^{\mathrm{c}}$ 390421162@qq.com
}

\begin{abstract}
Keywords: local charge-control meter; electricity price issuing; transparent task; meter reading
\end{abstract} mode; concentrator initiative.

\begin{abstract}
This paper describes in detail the principle and process of transparent task mode by comparing the traditional electricity-price issuing mode and the transparent task electricity-price issuing mode. Experimental results of the emulational platform district and the on-site platform district are integrated. This study validates that the actual effect and theoretical analysis of the electricity issuing program for the meter reading mode conform to each other completely. Furthermore, this study proves the feasibility of the transparent task mode in actual electricity information collection.
\end{abstract}

\section{Introduction}

With economic development and improvement of living standards, power consumption in production and daily life has increased rapidly, thereby bringing challenges to the safe and stable operations of power grids. Construction of the Strong Smart Grid [1] has been included in the national Twelfth Five-Year Plan. Further construction of the collection system and expanded application of the smart electricity meter are important aspects of constructing the Strong Smart Grid. Satisfactory construction of this grid is expected to benefit electric power customers and society in general.

The current electricity shortage in China cannot be relieved in a short period. To address this problem, orderly power consumption management based on a collection system is necessary. Since the implementation of tiered pricing for electricity, this policy has gained recognition in the market and among the parties concerned. The demand for multiple functions of the acquisition system has increased [2]. At present, local charge-control smart electricity meters [3] account for more than 90\% of smart meters in many regions, which indicates difficulty in electricity price issuing [4]. The electricity-price issuing work mode of the current electricity information collection system is transparent terminal transmission in which the main station of the system issues electricity prices through transparent terminal transmission. Actual feedback data show that this program is characterized by a low issuing success rate, large amount of time consumed, and task-issuing failure rate of approximately 30\%. The program requires manual debugging on the site and consumes significant manpower and material resources, especially in remote regions with sparse populations. In addition, it consumes significantly more time than automatic issuing [5].

\section{Differences between meter reading mode and traditional electricity-price issuing mode}

The design of electricity-price issuing systems has been improving in China [6], and power companies in different parts of the country are committed to developing a highly advanced mode.

When analyzing the original system, we found that the same communication process between the main station and the smart electricity meter (that is, the same communication channel) generally involves a daily collection success rate above $95 \%$ in the electricity information collection system throughout China. The average success rate of the communication channels of the collection system 
is high. Thus, theoretically, electricity price issuing can reach the level of meter-reading success rate if a reasonable approach is adopted.

The main purpose of the current price issuing mode is to achieve electricity price adjustment through real-time communication interaction of the main station of the system, terminal uplink and downlink modules, smart electricity meter, and other links.

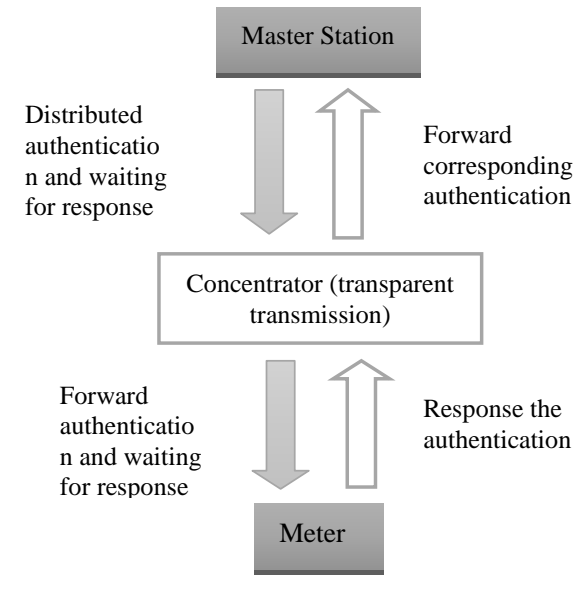

Figure 2. The traditional electricity distributed authentication process diagram

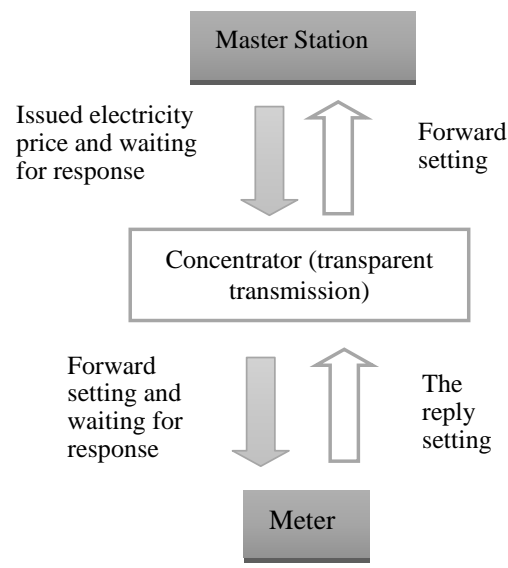

Figure 1. Traditional price issued price setting process diagram

First, the main station of the system issues an identity authentication command to the concentrator, which directly forwards the authentication command to the electricity energy meter. Then, both the main station and the concentrator are in the waiting state. After identity authentication, the electricity energy meter gives a response, which is reported to the main station step by step through the concentrator. The identity of the electricity energy meter is valid (Figure 2.1) only if the main station and the concentrator both receive the response within their respective timeout thresholds. The electricity price issuing setting is now issued from the main station to the electricity energy meter. Similar to the identity authentication command, the issuing setting is transparently transmitted through the concentrator (Figure 2.2). Similarly, a reply of the successful new electricity price setting of the electricity energy meter must be received within the communication timeout thresholds of the equipment that it goes through. Finally, it reaches the main station, which indicates successful completion of a traditional electricity issuing task. Therefore, the traditional electricity price issuing mode is a task mode that involves synchronous operation of multiple equipment. This condition indicates high requirements for real-time performance of the communication channel. The electricity price-issuing task fails in case of any of the four problems: main station timeout, concentrator timeout during reporting of identity authentication results, main station timeout, or concentrator timeout during reporting of setting results. In terms of hardware technology, neither GPRS/CDMA, which is commonly used in terminal uplink, nor narrow-band carrier wave channel for downlink is advantageous in real-time performance of a single communication. Therefore, the two types of hardware are set in one real-time task. The communication success probability is the product of their respective communication success rates $P_{i}\left(0<P_{i}<1\right)$, where $P_{i}=0$ is equipment failure, and $P_{i}=1$ indicates that $100 \%$ success rate does not exist in the long run. Neither of them is counted in a single communication (Formula 1). If a collector or another equipment is involved in the process, a probability multiplier is added, and the ultimate overall communication success rate $P$ decreases.

$$
\begin{aligned}
& P=\prod_{i=1}^{n} P_{i}, n \geq 2,0<P_{i}<1, \\
& P<\min \left\{P_{i}, i=1,2, \cdots, n\right\}, n \geq 2,0<P_{i}<1 .
\end{aligned}
$$

We suppose that $n=3$ if $P_{i}=90 \%$ and $i=1,2,3$. Thus, the success rate of a single communication is still as low as $P=0.9^{3}=72.9 \%$. As a result, the task requiring synchronization cannot be easily implemented. This factor is one of the technical reasons why the success rate of the 
original electricity price issuing mode task is only around $70 \%$.

Therefore, the disadvantage of the synchronization task [7] is as follows: To ensure real-time performance, the waiting time should not exceed the timeout thresholds of the equipment that a command goes through, so the communication quality of each channel must meet extremely high requirements. In addition, different equipment manufacturers set different work times for routing, and complicated conditions on the on-site platform district cannot meet the requirements completely. Based on the preceding analysis, the barrier to a high electricity price issuing mission success rate is that various response times must meet different timeout thresholds at the same time. If the system could be reconstructed and the asynchronous mode [8] adopted, that is, if the concentrator could share some issuing tasks for electricity price setting and save the results in the local equipment and the terminal temporarily instead of waiting for a real-time result response, and "asks" the concentrator for task performance results actively and intermittently, then the requirements for communication success rate of each channel would be significantly lowered, and the equipment would be able to repeatedly execute a task that fails temporarily until it succeeds. Thus, accidental communication failure would not influence the final electricity-price issuing results.

\section{Analysis of transparent task mode program}

Based on the requirements of electricity-price issuing work, power companies have organized relevant equipment software and hardware manufacturers to expand the transparent task protocol of Q/GDW 1376.1-2013, develop the electricity-price issuing program of the transparent task mode of the concentrator and the program function module of the main station, and conduct standard and regulatory concentrator program testing and laboratory verification. The transparent task plan has been finally determined as follows:

1) The main station issues identity authentication in batches to the concentrator, in which a transparent task queue is established.

2) The concentrator conducts electricity energy meter identity validation circularly in the platform district, and reports numbers of the electricity energy meters that have passed validation back to the main station. The task queue sequence deserves attention. The electricity energy meter that first passes validation is set first, and the next electricity energy meter that passes validation is set thereafter until all the electricity energy meters that pass identity validation are set or until identity validation expires.

3) The main station issues electricity price setting in batches to the concentrator based on identity validation results. In turn, the concentrator automatically conducts electricity price setting of electricity energy meters until successful completion. The process is exactly the same as that of concentrator meter reading.

4) To avoid timeout mechanism, the main station no longer waits for the concentrator reporting execution results. Instead, the main station actively inquires on the task execution results and confirms the completion of the electricity-price issuing task.

The concentrator directly initiates the transparent task plan. Synchronous interaction between the main station and the electricity energy meter is converted into asynchronous interaction. As a result, communication efficiency is increased and main station occupying time is reduced. During the entire transparent task process, a validity period only exists for identity authentication. This condition avoids task waiting indefinitely, unifies timeout thresholds in the entire system, and prevents problems caused by different routing timeout periods of different manufacturers. The priority strategy is adopted for tasks issued by different electricity energy meters so that daily collection is not influenced and transparent electricity price issuing tasks can be executed multiple times. Theoretically, electricity prices can be successfully issued to all the electricity energy meters that pass identity authentication. In the transparent task mode, compared with the traditional mode, routing plays the maximum role, and the electricity price issuing task is turned into a task similar to meter reading and then executed. The success rate of the electricity-price issuing task increases significantly to the level of the meter reading success rate, as verified in the on-site platform district. In the following section of this paper, the 
platform district validation results are described in detail.

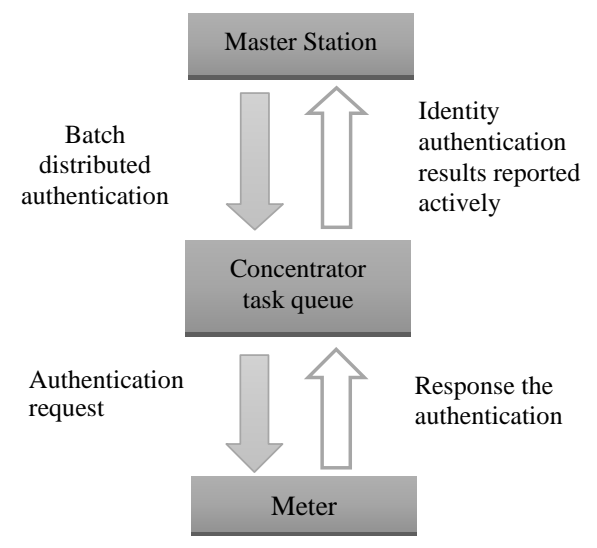

Figure 4. Transparent task electricity distributed authentication process diagram

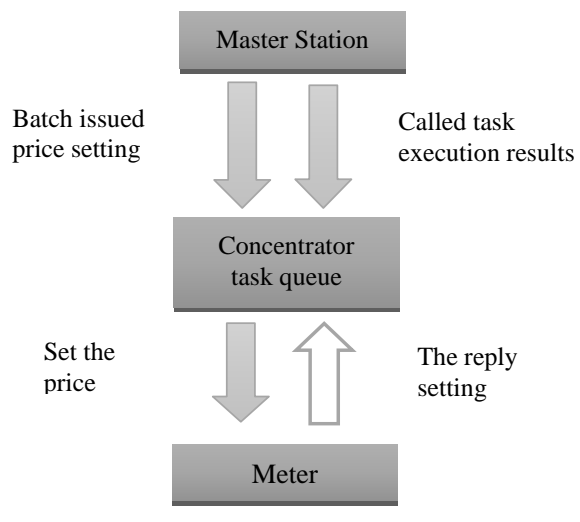

Figure 3. Transparent task issued price setting process diagram

The concentrator directly initiates the transparent task plan. Synchronous interaction between the main station and the electricity energy meter is converted into asynchronous interaction. As a result, communication efficiency is increased and main station occupying time is reduced. During the entire transparent task process, a validity period only exists for identity authentication. This condition avoids task waiting indefinitely, unifies timeout thresholds in the entire system, and prevents problems caused by different routing timeout periods of different manufacturers. The priority strategy is adopted for tasks issued by different electricity energy meters so that daily collection is not influenced and transparent electricity price issuing tasks can be executed multiple times. Theoretically, electricity prices can be successfully issued to all the electricity energy meters that pass identity authentication. In the transparent task mode, compared with the traditional mode, routing plays the maximum role, and the electricity price issuing task is turned into a task similar to meter reading and then executed. The success rate of the electricity-price issuing task increases significantly to the level of the meter reading success rate, as verified in the on-site platform district. In the following section of this paper, the platform district validation results are described in detail.

\section{Electricity price issuing platform district validation of transparent task mode}

Sigongxincun 011 (650131690) platform district of Urumqi Midong power supply station adopts the Topscomm carrier wave plan that consists of a total of 485 electricity energy meters of 15 meter factories, including 325 local charge-control meters. The meter reading success rate on the day before the experiment is $95.69 \%$. The platform district is a typical rural-urban fringe zone with complicated topology. The longest branch line is more than 1,000 meters long. The platform district is shown in Figure 5, and the experimental results are presented in Table 1. Long-distance measurement of the collection system and on-site verification (in which a total of 242 energy meters were verified) of Midong power supply company prove that all the issued electricity price results are correct.

In this experiment, only 311 local charge-control meters with successful meter reading are chosen, and issuing task failure occurs in 10 of these meters. Based on an analysis conducted by the test personnel, 7 electricity energy meters do not support long-distance electricity price issuing and should be excluded from the test base. In addition, communication failure occurs in 2 meters and identity authentication occurs in 1 meter. Therefore, the final corrected electricity price issuing success rate is $301 /(311-7)=99.01 \%$. The experimental results conform to the theoretical analysis presented in this paper. The on-site electricity-price issuing success rate is equivalent to the meter reading success rate. 


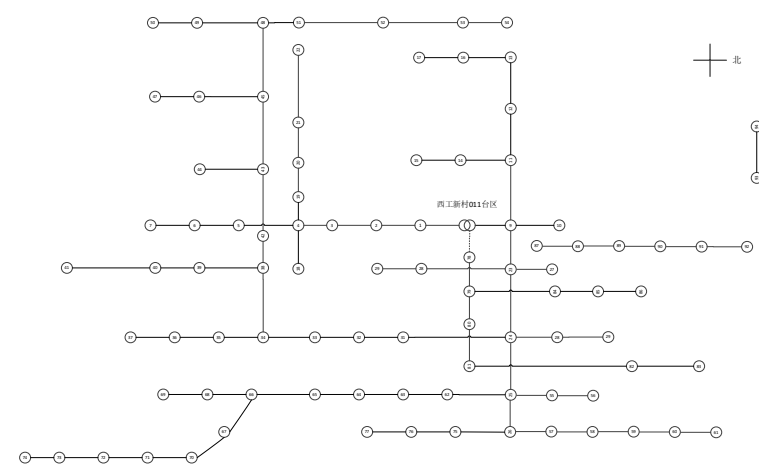

Figure 5. 011 area topological diagram

Table 1 The simulation area transparent task model electricity distributed scheme test results

\begin{tabular}{|c|l|}
\hline Electricity price issued last & start time \\
& $2015-06-22$ 01:00 \\
& completion time \\
& $2015-06-22$ 15:10 \\
& After took about 14 hours (including zero meter reading \\
& time and electricity price issued time) \\
\hline total meter number of Electricity & 311 \\
price issued & \\
\hline Successful meter number & 301 \\
\hline Failure meter number & 10 \\
\hline success rate & $96.78 \%$ \\
\hline
\end{tabular}

\section{Conclusions}

After Electricity price issuing by local charge-control electricity energy meters in the transparent task mode theoretically avoids the high requirement of real-time performance of the channel because of electricity synchronization of the traditional electricity-price issuing mode. Experiments in the emulational platform district and the on-site platform district prove that this method increases the electricity-price issuing success rate. The transparent task mode improves the compatibility of the collection system for different terminals provided by different manufacturers, eliminates the influence of different timeout settings of different equipment on the task, and solves the problem of frequent upgrading that results from equipment compatibility. In addition, the method requires no new hardware input and only needs concentrator upgrading and the main station software of the system. Thus, the method entails less improvement cost.

By adopting the electricity price issuing mode with high success rate, power companies can expand their business conveniently. For example, clock adjustment of an electricity energy meter, residual amount modification, and other tasks can be executed in a manner similar to the transparent task. This condition indicates greater possibilities for further construction of the Strong Smart Grid in the future.

\section{References}

[1] Li Xingyuan, Wei wei, Wang Yuhong, Mu Zilong, Gu Wei, "Study on the development and technology of strong smart grid (Chinese)", POWER SYSTEM PROTECTION AND CONTROL, 2009, 37(17):1-7J.

[2] Li Jinsha, Zheng Fangqin, "Optimize the remote fee charged business booster marketing file to upgrade (Chinese)”, Rural Electrician, 2016(1).

[3] Tao Peng, Zhang Yan, Cao Huimin, Characteristics and parameter setting analysis on local control smart electricity meter (Chinese)”, Hebei electric power, 2012(1):8-9.

[4] Li Ruijie, Ma Xinyuan, "Analyses the local fee charged watt-hour meter owe the reason and solution (Chinese)”, power supply technologies and applications, 2014. 
[5] Lin Nvgui, Mo Sangbi, "Study on the Power Supply Enterprise's Selection on the Smart Electric Meter (Chinese)”, Journal of Minjiang University, 2013, 34(1):40-43.

[6] Fu Feng, Wang Jun, Tao Xiaofeng, “The electricity distributed scheduling system based on electricity information acquisition design (Chinese)”, Symposion of China institute of electrical engineering professional committee of power system automation, 2012

[7] GK Manacher, “Production and Stabilization of Real-Time Task Schedules”, Journal of the Acm, 1967, 14(3):439-465.

[8] H Kaiser, T Heller, D Bourgeois, D Fey, Higher-level Parallelization for Local and Distributed Asynchronous Task-Based Programming, First International Workshop on Extreme Scale Programming Models and Middleware , At Austin, Texas, USA, 2015 